Volume 26 (2019) 131-144

DOI: $10.24330 /$ ieja. 587018

\title{
ON FINITE DIMENSIONAL ALGEBRAS WHICH ARE SUMS OF TWO SUBALGEBRAS
}

\author{
M. Tamer Koşan and Jan Žemlička \\ Received: 1 January 2019; Revised: 27 March 2019; Accepted: 2 April 2019 \\ Communicated by Abdullah Harmancı
}

\begin{abstract}
In this paper, we give a general method of the construction of a 3-dimensional associative algebra $R$ over an arbitrary field $F$ that is a sum of two subalgebras $R_{1}$ and $R_{2}$ (i.e. $R=R_{1}+R_{2}$ ).
\end{abstract}

Mathematics Subject Classification (2010): 16N40, 16R10, 16S36, 16W60

Keywords: Sum of rings, sum of algebras

\section{Introduction}

Let $R$ be an associative ring. Writing $R=R_{1}+R_{2}$, we mean that $R_{1}$ and $R_{2}$ are subrings of $R$ and, for every $r \in R$, there exist $r_{1} \in R_{1}$ and $r_{2} \in R_{2}$ such that $r=r_{1}+r_{2}$.

Many authors studied problems and relationships among properties of $R_{1}, R_{2}$ and $R$ (see [4], [5], [7], [8], [10], [11], [12], [13]). For instance, the question "is $R$ nil provided that $R_{1}^{2}=0$ and $R_{2}$ is nil?" is equivalent to the famous Köthe's nil ideal problem (see [14], [15] and [16]). Let us mention three other open problems in this area.

Suppose that $R_{1}$ and $R_{2}$ are rings satisfying a polynomial identity. Does $R$ satisfies a polynomial identity ([1])? The answer to this problem is known in several particular cases (cf. [3], [7], [8], [9], [10], [11], [12], [13]), and a full positive answer in [12].

It is known [14] that if both $R_{1}$ and $R_{2}$ are nilpotent rings, then so is $R$. It is also known that there exists a function $f(n, m)$ such that if $R_{1}^{n}=0$ and $R_{2}^{m}=0$, then $R^{f(n, m)}=0$. However the best such a function is still unknown. It is conjectured that $f(m, n)=m n$.

For the last problem, suppose that $R_{1}$ is nil and $R_{2}$ is reduced (it has no nonzero nilpotent elements). Is $R_{1}$ an ideal of $R$ ? The answer to this question is known to be "yes" in many particular cases [13], but in general, is still unknown. 
We remark that a general method of constructing rings which are sums of two subrings was invited by Kelarev [6]. As we mentioned before, all these problems, on their substantial parts, concern due structure of rings which are sums of two subrings. This is particularly clear for the question 3. Note that it also concerns questions first and second. Namely, if $R$ is a semiprime ring which satisfies a polynomial identity, then the ring of Ore extension of $R$ is isomorphic to a direct sum of matrix rings over division rings. Thus, when studying whether of $R=R_{1}+R_{2}$, and each $R_{i}$ satisfies a polynomial identity implies $R$ satisfies such an identity in the case of semiprime $R$ to study the structure of subrings of matrix rings of two subrings. Here, we also note that, Bokut [2] proved that every algebra over a field can be embedded into a simple algebra which is a sum of three nilpotent subalgebras. In view of these two facts, we will a general method of constructing 2 and 3 dimensional algebras over a field $F$ which are sums of to subalgebras with respect to the isomorphisms. This general method may give us to describe semiprime rings (even finite dimensional algebras) as a sum of two subrings.

\section{Some general results}

Suppose that $F$ is a field and $R$ is a finite dimensional (non-unitary) associative $F$-algebra. Put $A:=F \times R$ and define binary operation + and $\cdot$ on $A$ by the rule

$$
\left(f_{1}, r_{1}\right)+\left(f_{2}, r_{2}\right)=\left(f_{1}+f_{2}, r_{1}+r_{2}\right)
$$

and

$$
\left(f_{1}, r_{1}\right) \cdot\left(f_{2}, r_{2}\right)=\left(f_{1} \cdot f_{2}, f_{1} r_{2}+f_{2} r_{1}+r_{1} r_{2}\right)
$$

where $f_{1}, f_{2} \in F$ and $r_{1}, r_{2} \in R$. It is easy to say that $A$ is an $F$-vector space and we may identify elements $r \in R$ with elements $(0, r)$ in $A$.

Proposition 2.1. $A$ is a finite dimensional $F$-algebra with a unit and $R$ is a two sided ideal of $A$ such that $\operatorname{dim}_{F}(A / R)=1$. Moreover, $A / R \cong F$.

Proof. Since $A$ is a vector space over $F$, it is easy to see that

$$
\begin{aligned}
f(a, b) & =(f, 0) \cdot(a, b) \\
& =(f a, f b) \\
& =(a, b) \cdot(f, 0),
\end{aligned}
$$

which shows that $(1,0)$ is a unit. 
For each $\left(f_{i}, r_{i}\right) \in A$, we get

$$
\begin{aligned}
\left(f_{1}, r_{1}\right)\left[\left(f_{2}, r_{2}\right)\left(f_{3}, r_{3}\right)\right] & =\left(f_{1}, r_{1}\right)\left(f_{2} f_{3}, f_{2} r_{3}+f_{3} r_{2}+r_{2} r_{3}\right) \\
& =\left(f_{1} f_{2} f_{3}, f_{1} f_{2} r_{3}+f_{1} f_{3} r_{2}+f_{1} r_{2} r_{3}+f_{2} f_{3} r_{1}+f_{2} r_{1} r_{3}\right. \\
& \left.+f_{3} r_{1} r_{2}+r_{1} r_{2} r_{3}\right) \\
& =\left(f_{1} f_{2}, f_{1} r_{2}+f_{2} r_{1}+r_{1} r_{2}\right)\left(f_{3}, r_{3}\right) \\
& =\left[\left(f_{1}, r_{1}\right)\left(f_{2}, r_{2}\right)\right]\left(f_{3}, r_{3}\right) .
\end{aligned}
$$

Similarly

$$
\begin{aligned}
\left(f_{1}, r_{1}\right)\left[\left(f_{2}+f_{3}, r_{2}+r_{3}\right)\right] & =\left(f_{1} f_{2}+f_{1} f_{3}, f_{1} r_{2}+f_{1} r_{3}+f_{2} r_{1}+f_{3} r_{1}+r_{1} r_{2}+r_{1} r_{3}\right) \\
& =\left(f_{1}, r_{1}\right)\left(f_{2}, r_{2}\right)+\left(f_{1}, r_{1}\right)\left(f_{3}, r_{3}\right)
\end{aligned}
$$

and

$$
\left[\left(f_{2}+f_{3}, r_{2}+r_{3}\right)\right]\left(f_{1}, r_{1}\right)=\left(f_{2}, r_{2}\right)\left(f_{1}, r_{1}\right)+\left(f_{3}, r_{3}\right)\left(f_{1}, r_{1}\right) .
$$

Clearly, $R$ is $F$-subspace of $A$ such that $\operatorname{dim}_{F}(A)=\operatorname{dim}_{F}(R)+1$ and

$$
\begin{aligned}
& (f, r)(0, s)=(0, f s+r s) \\
& (0, s)(f, r)=(0, s f+s r)
\end{aligned}
$$

for every $(f, r) \in A$ and $s \in R$. Hence $R$ is a two-sided ideal of $A$. Finally, $A / R \cong F$.

For an algebra $R$, the symbol $R^{*}$ denotes $R$ with an identity adjoined.

Theorem 2.2. If $R$ does not contain a unit, then it is an ideal of finite dimensional $F$-algebra $A$ such that $\operatorname{dim}_{F}(A)=\operatorname{dim}_{F}(R)+1$, i.e. there exists a complete orthogonal set of primitive idempotents $e_{0}, \ldots, e_{m}$ such that $e_{0} \in A \backslash R, e_{1}, \ldots, e_{m} \in R$, $R=A\left(1-e_{0}\right) A$ and $\operatorname{dim}_{F}\left(e_{0} A / e_{0} J(A)\right)=1$.

Proof. The first part follows from Proposition 2.1. The rest of the claim follows from [17, Proposition VIII.4.1], since every orthogonal set of idempotents of $R+$ $J(A) / J(A)$ can be lifted to an orthogonal set of idempotents of $R$.

Remark 2.3. Fix $A$ from Theorem 2.2 if $R$ does not contain a unit and put $A=R$ otherwise. We know that there exists a complete orthogonal set of primitive idempotents $e_{0}, \ldots, e_{m}$ of $A$ such that $e_{1}, \ldots, e_{m} \in R$ and $\operatorname{dim}_{F}\left(e_{0} A / e_{0} J(A)\right)=1$ if $A \neq R$. Then $m<\operatorname{dim}_{F} R$.

Proof. Obviously, $m \leq \operatorname{dim}_{F} R \leq \operatorname{dim}_{F} A$ and, moreover, $m \neq \operatorname{dim}_{F} R$ if $R=A$. Let $R \neq A$. If $e_{0} A$ is simple, then $1-e_{0} \in R$ is a unit of $R$, a contradiction. Thus $\operatorname{dim}_{F}\left(e_{0} A\right)>1$, hence $m<\operatorname{dim}_{F} R$. 


\section{Two structure theorems}

The following observation is easy.

Lemma 3.1. If $0 \neq R_{1}$ and $0 \neq R_{2}$ are proper subalgebras of the algebra $R$ over a field $F$ and $\operatorname{dim}_{F} R=2$, then $\operatorname{dim}_{F} R_{1}=1=\operatorname{dim}_{F} R_{2}$.

Assume that $\operatorname{dim}_{F} R=2$ such that $R$ is a sum of subalgebras $R_{1}$ and $R_{2}$. By Lemma 3.1, we may assume that $\operatorname{dim}_{F} R_{1}=1=\operatorname{dim}_{F} R_{2}$. Let $R_{1}=F e$ and $R_{2}=F f$, where $e^{2}=e \in R$ and $f^{2}=f \in R$, or $e^{2}=0$ or $f^{2}=0$ if $R_{1} \cong F^{0}$ or $R_{2} \cong F^{0}$, where $F^{0}$ denotes $F$ with zero multiplication. Then we have the following cases on $R_{1}$ (or $R_{2}$ ).

* If $R_{1}^{2}=0$, then $R_{1} \cong F^{0}$. Hence we have either $R_{2} \cong F^{0}$ or $R_{2} \cong F$.

** If $R_{1}^{2} \neq 0$, then $R_{1} \cong F$. Hence we have either $R_{2} \cong F^{0}$ or $R_{2} \cong F$.

Case 1. Let $R_{1}^{2}=0, R_{1} \cong F^{0}$ and $R_{2} \cong F^{0}$. For $\alpha \in R_{1}$ and $\beta \in R_{2}$, write

$$
e f=\alpha e+\beta f
$$

Note that, in this case, $e^{2}=0=f^{2}$. Multiplying the equation (1) by $f$ on the right, we get $\alpha e f=0$ which implies $\alpha=0$ or ef $=0$. If $\alpha=0$, we get $e f=\beta f$. Thus $\beta e f=0$. It follows $e f=0$. Similarly, we obtain that $f e=0$. Now it is enough to take $R=F^{0} \times F^{0}, e=(1,0)$ and $f=(0,1)$.

Case 2. Let $R_{1}^{2} \neq 0, R_{1} \cong F$ and $R_{2} \cong F$. Then, for $\alpha \in R_{1}$ and $\beta \in R_{2}$, we get again the equation (1). Multiplying the equation (1) by $e$ on the left, we get

$$
e f=\alpha e+\beta e f
$$

Now, $0=\beta(e f-f)$ implies either $\beta=0$ or $e f=f$. If $\beta=0$, then, by equation (1), ef $=\alpha e$. Since $\alpha e=e f=e f^{2}=\alpha e f=\alpha^{2} e$, we get either $\alpha=1$ or $\alpha=0$. By equation (1), if $\beta=0$ and $\alpha=0$, then we have $e f=0$ and so $f e=0$, if $\beta=0$ and $\alpha=1$ then ef $=e$. Therefore $e f \in\{0, e, f\}$.

By similar reasoning as above we can obtain that $f e \in\{0, e, f\}$. Assume that ef $=0$. Multiplying the equation (3) by $f$ on the right, we have $\beta_{1} f=0$. So $\beta_{1}=0$. Consequently $0=e f e=\alpha_{1} e$ which implies $\alpha_{1}=0$. Hence $f e=0$. We can take $R=F \times F$ and $e=(1,0), f=(0,1)$.

Let $e f=f=f e$. So $e$ is an identity element of $R$. Therefore $e-f$ and $f$ are orthogonal idempotents of $R$. Consequently $R=F \times F$ and we can take $e=(1,1)$ and $f=(0,1)$. The case where $e f=e=f e$ can be treated analogously. 
In the case where $e f=f$ and $f e=e$, consider $R=\left(\begin{array}{cc}F & F \\ 0 & 0\end{array}\right)$, where $e=\left(\begin{array}{ll}1 & 0 \\ 0 & 0\end{array}\right)$ and $f=\left(\begin{array}{ll}1 & 1 \\ 0 & 0\end{array}\right)$

Analogously, if $e f=e$ and $f e=f$, let $R=\left(\begin{array}{ll}F & 0 \\ F & 0\end{array}\right)$, where $e=\left(\begin{array}{ll}1 & 0 \\ 0 & 0\end{array}\right)$ and $f=\left(\begin{array}{ll}1 & 0 \\ 1 & 0\end{array}\right)$.

Case 3. Let $R_{1}^{2} \neq 0, R_{1} \cong F$ and $R_{2} \cong F^{0}$. Multiplying the equation (1) by $f$ on the right, we get $0=\alpha e f$ which implies either $\alpha=0$ or ef $=0$.

If $\alpha=0$, then $e f=\beta f$ by equation (1). Now $e f=e^{2} f=e e f=e \beta f=\beta e f=$ $\beta^{2} f$ which implies either $\beta=0$ or $\beta=1$. Clearly, if $\beta=0$ then ef $=0$ and if $\beta=1$, then ef $=f$.

Finally, multiplying the equation (3) by $f$ on the right, we get $\alpha_{1} e f=f e f$. So $\alpha_{1} e=0$ because $e f=f$ or $e f=0$. Hence $f e=\beta_{1} f=\beta_{1}^{2} f$. It follows that $\beta_{1}=1$ or $\beta_{1}=0$. Consequently, $f e=0$ or $f e=f$.

In the case where ef $=f e=0$, we take $R=F \times F^{0}$, where $e=(1,0)$ and $f=(0,1)$. Note that if $R=\left(\begin{array}{cc}F & F \\ 0 & 0\end{array}\right), e=\left(\begin{array}{ll}1 & 0 \\ 0 & 0\end{array}\right)$ and $f=\left(\begin{array}{ll}0 & 0 \\ 1 & 0\end{array}\right)$, then ef $=0$ and $f e=f$.

Finally, let $R=\left(F^{0}\right)^{*}, e=(1,0)$ and $f=(0,1)$. Clearly, ef $=f=f e$.

As a result of above calculations, we have the following observation.

Theorem 3.2. Assume that $\operatorname{dim}_{F} R=2$ and $R=R_{1}+R_{2}$ is a sum of proper subalgebras $R_{1}$ and $R_{2}$. Then we have the following cases.

(I) If $R_{1} \cong F^{0}$ and $R_{2} \cong F^{0}$, then $R=F^{0} \times F^{0}$.

(II) If $R_{1} \cong F$ and $R_{2} \cong F$, then one of the following cases holds true:

(1) $R=F \times F$,

(2) $R=\left(\begin{array}{ll}F & F \\ 0 & 0\end{array}\right)$,

(3) $R=\left(\begin{array}{ll}F & 0 \\ F & 0\end{array}\right)$.

(III) If $R_{1} \cong F$ and $R_{2} \cong F^{0}$, then one of the following cases holds true:

(1) $R=F \times F^{0}$,

(2) $R=\left(\begin{array}{ll}F & F \\ 0 & 0\end{array}\right)$, 

(3) $R=\left(\begin{array}{ll}F & 0 \\ F & 0\end{array}\right)$,
(4) $R=\left(F^{0}\right)^{*}$.

In the next Theorem, we will use the following.

Remark 3.3. Let $M$ be an $A$-A-bimodule, denote $\mu(M)$ a matrix $\mu(M)=\left(d_{i j}\right)$ where $d_{i j}=\operatorname{dim}_{F}\left(e_{i} M e_{j}\right)$. Denote by $S$ a ring of polynomials in two noncommuting variables $x_{1}, x_{2}$ over $F$. If $\mathbf{A}=\left(a_{k l}\right)$ is a matrix where $a_{k l} \in F, 1 \leq k, l \leq 2$, then define

$S(\mathbf{A})=S /\left\langle a_{22} x_{1}^{2}-a_{11} x_{2}^{2}, a_{21} x_{1} x_{2}-a_{12} x_{2} x_{1}, a_{22} x_{1} x_{2}-a_{12} x_{2}^{2}, a_{22} x_{2} x_{1}-a_{21} x_{2}^{2}, x_{1}^{3}, x_{2}^{3}\right\rangle$, where $a_{11}, a_{12}, a_{21}, a_{22} \neq 0$. Moreover,

$$
S(\mathbf{A})=S /\left\langle x_{1}^{2}, a_{21} x_{1} x_{2}-a_{12} x_{2} x_{1}, a_{22} x_{1} x_{2}-a_{12} x_{2}^{2}, a_{22} x_{2} x_{1}-a_{21} x_{2}^{2}, x_{2}^{3}\right\rangle
$$

if $a_{11}=0$,

$$
S(\mathbf{A})=S /\left\langle x_{1}^{2}, x_{1} x_{2}, a_{22} x_{2} x_{1}-a_{21} x_{2}^{2}, x_{2}^{3}\right\rangle
$$

if $a_{11}=a_{12}=0$,

$$
S(\mathbf{A})=S /\left\langle x_{1}^{2}, x_{1} x_{2}, x_{2} x_{1}, x_{2}^{3}\right\rangle
$$

if $a_{11}=a_{12}=a_{21}=0$. Analogously we define $S(\mathbf{A})$ in the case $a_{11}=a_{22}=0$ or $a_{11}=a_{12}=a_{22}=0$.

Assume that $\operatorname{dim}_{F} R=3$ such that $R$ is a sum of subalgebras order of $R_{1}$ and $R_{2}$.

Theorem 3.4. Assume that $\operatorname{dim}_{F} R=3$ such that $R$ is a sum of subalgebras order of $R_{1}$ and $R_{2}$.

(I) Let $m=0$ and $R=A$. Then one of the following cases holds true:

(a) If $J(R)=0$, then $R \cong K$, where $K$ is a field of $F$-dimension 3 and either $R_{1}=R$ or $R_{2}=R$.

(b) If $R / J(R) \cong F$ and $J^{2}(R)=0$, then $R \cong F\left[x_{1}, x_{2}\right] /\left\langle x_{1}^{2}, x_{1} x_{2}, x_{2}^{2}\right\rangle$ and

(1) $R_{1}=R$ and $R_{2}$ is an arbitrary subalgebra of $R$.

(2) $R_{1} \cong F_{0} \times F_{0}$ and $R_{2} \cong F$.

(3) $R_{1} \cong F[x] /\left\langle x^{2}\right\rangle$ and $R_{2} \cong F_{0}$.

(4) $R_{1} \cong F[x] /\left\langle x^{2}\right\rangle$ and $R_{2} \cong F_{0} \times F_{0}$.

(5) $R_{1} \cong R_{2} \cong F[x] /\left\langle x^{2}\right\rangle$.

(c) If $R / J(R) \cong F$ and $J^{2}(R) \neq 0$, then $R \cong F[x] /\left\langle x^{3}\right\rangle$ and

(6) $R_{1}=R$ and $R_{2}$ is an arbitrary subalgebra of $R$,

(7) $R_{1} \cong x F[x] /\left\langle x^{3}\right\rangle$ and $R_{2} \cong F$, 
ON FINITE DIMENSIONAL ALGEBRAS WHICH ARE SUMS OF TWO SUBALGEBRAS 137

(8) $R_{1} \cong x F[x] /\left\langle x^{3}\right\rangle, R_{2} \cong F[x] /\left\langle x^{2}\right\rangle$, where $F[x] /\left\langle x^{2}\right\rangle \cong F+$ $x^{2} F \subset R$.

Furthermore, all the cases in (1) are commutative.

(II) Let $m=1$ and $R=A$. Then one of the following cases holds true:

(a) If $J(R)=0$, then $R \cong F \times G$ where $G$ is a field of $F$-dimension 2 and

(1) $R_{1}=R$ and $R_{2}$ is an arbitrary subalgebra of $R$,

(2) $R_{1} \cong G$ and $R_{2} \cong F$,

(3) $R_{1} \cong G$ and $R_{2} \cong F \times F$.

(b) If $J(R) \neq 0$, then $R \cong\left(\begin{array}{ll}F & F \\ 0 & F\end{array}\right)$ and we have one of the following cases.

(4) $R_{1}=R$ and $R_{2}$ is an arbitrary subalgebra of $R$,

(5) $R_{1} \cong F^{2}$ and $R_{2} \cong F_{0}$,

(6) $R_{1} \cong\left(\begin{array}{cc}F & F \\ 0 & 0\end{array}\right)$ and $R_{2} \cong F$,

(7) $R_{1} \cong\left(\begin{array}{ll}0 & F \\ 0 & F\end{array}\right)$ and $R_{2} \cong F$,

(8) $R_{1} \cong F^{2}$ and $R_{2} \cong\left(\begin{array}{ll}0 & F \\ 0 & F\end{array}\right)$,

(9) $R_{1} \cong F^{2}$ and $R_{2} \cong\left(\begin{array}{cc}F & F \\ 0 & 0\end{array}\right)$,

(10) $R_{1} \cong\left(\begin{array}{cc}F & F \\ 0 & 0\end{array}\right)$ and $R_{2} \cong\left(\begin{array}{ll}0 & F \\ 0 & F\end{array}\right)$.

(III) Let $m=2$ and $R=A$. Then $R \cong F \times F \times F$ whenever $m=2$ and so we have one of the following cases.

(a) $R_{1}=R$ and $R_{2}$ is an arbitrary (semisimple) subalgebra of $R$,

(b) $R_{1} \cong F \times F$ and $R_{2} \cong F$,

(c) $R_{1} \cong R_{2} \cong F \times F$.

(IV) Let $m=0$ and $R \neq A$. If $J^{2}(R)=0$, then $R \cong F_{0}^{3}$, and so $R_{1} \cong F_{0}^{k}$ and $R_{2} \cong F_{0}^{l}$ for $k, l \leq 3$. We have one of the following cases.

(a) If $J^{2}(R)=0$, then $R \cong F_{0}^{3}$, and so $R_{1} \cong F_{0}^{k}$ and $R_{2} \cong F_{0}^{l}$ for $k, l \leq 3$.

(b) If $J^{2}(R) \neq 0, J^{3}(R)=0$ and $\operatorname{dim}_{F}\left(J(R) / J^{2}(R)\right)>\operatorname{dim}_{F}\left(J^{2}(R) / J^{3}(R)\right)$, then $R \cong\left\{x_{1}, x_{2}\right\} S(\mathbf{A})$ and

(1) $R_{1}=R$ and $R_{2}$ is an arbitrary subalgebra of $R$,

(2) $R_{1} \cong x F[x] /\left\langle x^{3}\right\rangle$ and $R_{2} \cong F_{0}$,

(3) $R_{1} \cong F_{0}^{2}$ and $R_{2} \cong F_{0}$,

(4) $R_{1} \cong R_{2} \cong x F[x] /\left\langle x^{3}\right\rangle$, 
(5) $R_{1} \cong x F[x] /\left\langle x^{3}\right\rangle$ and $R_{2} \cong F_{0}^{2}$,

(6) $R_{1} \cong F_{0}^{2}$ and $R_{2} \cong F_{0}^{2}$.

(c) If $J^{3}(R) \neq 0$, then $R=R_{1} \cong x F[x] /\left\langle x^{4}\right\rangle$ and $R_{2}$ is an arbitrary (chain) subalgebra of $R$.

(V) Let $m=1$ and $R \neq A$. Then $A / J(R) \cong F \times G$ where $G$ is a field and $\operatorname{dim}_{F}(G) \leq 2$. Let $G \cong F$. We have one of the following cases.

(a) If $J^{2}(R)=0$ and $\mu(J(R))=\left(\begin{array}{ll}1 & 0 \\ 0 & 1\end{array}\right)$, then $R \cong F_{0} \times F[x] /\left\langle x^{2}\right\rangle$ and

(1) $R_{1}=R$ and $R_{2}$ is an arbitrary subalgebra of $R$,

(2) $R_{1} \cong F[x] /\left\langle x^{2}\right\rangle$ and $R_{2} \cong F_{0}$,

(3) $R_{1} \cong F_{0} \times F$ and $R_{2} \cong F_{0}$,

(4) $R_{1} \cong F[x] /\left\langle x^{2}\right\rangle$ and $R_{2} \cong F_{0}^{2}$,

(5) $R_{1} \cong F[x] /\left\langle x^{2}\right\rangle$ and $R_{2} \cong F_{0} \times F$.

(b) If $J^{2}(R)=0$ and $\mu(J(R))=\left(\begin{array}{ll}0 & 1 \\ 0 & 1\end{array}\right)$, then $R \cong\left\{\left(\begin{array}{lll}0 & 0 & b \\ 0 & a & c \\ 0 & 0 & a\end{array}\right) \mid a, b, c \in F\right\}$ and

(6) $R_{1}=R$ and $R_{2}$ is an arbitrary subalgebra of $R$,

(7) $R_{1}=J(R) \cong F_{0}^{2}$ and $R_{2} \cong F$,

(8) $R_{1}=\left(\begin{array}{ll}F & 0 \\ F & 0\end{array}\right)$ and $R_{2} \cong F_{0}$,

(9) $R_{1} \cong F[x] /\left\langle x^{2}\right\rangle$ and $R_{2} \cong F_{0}$,

(10) $R_{1}=J(R) \cong F_{0}^{2}$ and $R_{2} \cong F[x] /\left\langle x^{2}\right\rangle$,

(11) $R_{1}=J(R) \cong F_{0}^{2}$ and $R_{2} \cong\left(\begin{array}{ll}F & 0 \\ F & 0\end{array}\right)$,

(12) $R_{1} \cong\left(\begin{array}{ll}F & 0 \\ F & 0\end{array}\right)$ and $R_{2} \cong F[x] /\left\langle x^{2}\right\rangle$.

(c) If $J^{2}(R)=0$ and $\mu(J(R))=\left(\begin{array}{ll}0 & 0 \\ 1 & 1\end{array}\right)$, then $R \cong\left\{\left(\begin{array}{lll}0 & 0 & 0 \\ 0 & a & 0 \\ b & c & a\end{array}\right) \mid a, b, c \in F\right\}$. In fact, this case is antiisomorphic to the case (b) (i.e. it has the structure of $R^{o p}$ ).

(d) If $J^{2}(R)=0$ and $\mu(J(R))=\left(\begin{array}{ll}1 & 0 \\ 1 & 0\end{array}\right)$, then $R \cong\left(\begin{array}{ccc}F & 0 & F \\ 0 & 0 & F \\ 0 & 0 & 0\end{array}\right)$ and

(13) $R_{1}=R$ and $R_{2}$ is an arbitrary subalgebra of $R$,

(14) $R_{1} \cong F_{0}^{2}$ and $R_{2} \cong F$, 
(15) $R_{1}=\left(\begin{array}{ll}F & 0 \\ F & 0\end{array}\right)$ and $R_{2} \cong F_{0}$,

(16) $R_{1} \cong F \times F_{0}$ and $R_{2} \cong F_{0}$,

(17) $R_{1}=J(R) \cong F_{0}^{2}$ and $R_{2} \cong F \times F_{0}$,

(18) $R_{1}=J(R) \cong F_{0}^{2}$ and $R_{2} \cong\left(\begin{array}{ll}F & 0 \\ F & 0\end{array}\right)$,

(19) $R_{1} \cong\left(\begin{array}{ll}F & 0 \\ F & 0\end{array}\right)$ and $R_{2} \cong F \times F_{0}$.

(e) If $J^{2}(R)=0$ and $\mu(J(R))=\left(\begin{array}{ll}1 & 1 \\ 0 & 0\end{array}\right)$, then $R \cong\left(\begin{array}{ccc}F & 0 & 0 \\ 0 & 0 & 0 \\ F & F & 0\end{array}\right)$.

(f) If $J^{2}(R)=0$ and $\mu(J(R))=\left(\begin{array}{ll}0 & 1 \\ 1 & 0\end{array}\right)$, then $R \cong\left\{\left(\begin{array}{lll}a & b & 0 \\ 0 & 0 & c \\ 0 & 0 & a\end{array}\right) \mid a, b, c \in F\right\}$

and

(20) $R_{1}=R$ and $R_{2}$ is an arbitrary subalgebra of $R$,

(21) $R_{1} \cong F_{0}^{2}$ and $R_{2} \cong F$,

(22) $R_{1}=\left(\begin{array}{ll}F & 0 \\ F & 0\end{array}\right)$ and $R_{2} \cong F_{0}$,

(23) $R_{1} \cong\left(\begin{array}{cc}F & F \\ 0 & 0\end{array}\right)$ and $R_{2} \cong F_{0}$,

(24) $R_{1}=J(R) \cong F_{0}^{2}$ and $R_{2} \cong\left(\begin{array}{cc}F & F \\ 0 & 0\end{array}\right)$,

(25) $R_{1}=J(R) \cong F_{0}^{2}$ and $R_{2} \cong\left(\begin{array}{ll}F & 0 \\ F & 0\end{array}\right)$,

(26) $R_{1} \cong\left(\begin{array}{ll}F & 0 \\ F & 0\end{array}\right)$ and $R_{2} \cong\left(\begin{array}{cc}F & F \\ 0 & 0\end{array}\right)$.

(g) If $\operatorname{dim}_{F} G=2$, then $J(R)$ is not a $G$-module, hence $R \cong G \times F_{0}$ and

(27) $R_{1}=R$ and $R_{2}$ is an arbitrary subalgebra of $R$,

(28) $R_{1} \cong G$ and $R_{2} \cong F_{0}$,

(29) $R_{1} \cong G$ and $R_{2} \cong F \times F_{0}$.

(h) If $J^{2}(R) \neq 0$, then $R \cong F \times\left(x F[x] /\left\langle x^{3}\right\rangle\right)$ and

(30) $R_{1}=R$ and $R_{2}$ is an arbitrary subalgebra of $R$,

(31) $R_{1} \cong x F[x] /\left\langle x^{3}\right\rangle$ and $R_{2} \cong F$,

(32) $R_{1} \cong x F[x] /\left\langle x^{3}\right\rangle$ and $R_{2} \cong F \times F_{0}$. 
(VI) Let $m=2$ and $R \neq A$. Then $A / J(R) \cong F^{3}$ and we have one of the following cases.

(a) $R \cong F^{2} \times F_{0}$ and

(1) $R_{1}=R$ and $R_{2}$ is an arbitrary subalgebra of $R$,

(2) $R_{1} \cong F^{2}$ and $R_{2} \cong F_{0}$,

(3) $R_{1} \cong F \times F_{0}$ and $R_{2} \cong F$,

(4) $R_{1} \cong F^{2}$ and $R_{2} \cong F \times F_{0}$.

(b) $R \cong F \times\left(\begin{array}{ll}F & 0 \\ F & 0\end{array}\right)$ and

(5) $R_{1}=R$ and $R_{2}$ is an arbitrary subalgebra of $R$,

(6) $R_{1} \cong F^{2}$ and $R_{2} \cong F_{0}$,

(7) $R_{1} \cong\left(\begin{array}{ll}F & 0 \\ F & 0\end{array}\right)$ and $R_{2} \cong F$,

(8) $R_{1} \cong F \times F_{0}$ and $R_{2} \cong F$,

(9) $R_{1} \cong F^{2}$ and $R_{2} \cong F \times F_{0}$,

(10) $R_{1} \cong F^{2}$ and $R_{2} \cong\left(\begin{array}{ll}F & 0 \\ F & 0\end{array}\right)$,

(11) $R_{1} \cong\left(\begin{array}{ll}F & 0 \\ F & 0\end{array}\right)$ and $R_{2} \cong F \times F_{0}$.

(c) $R \cong F \times\left(\begin{array}{ll}F & F \\ 0 & 0\end{array}\right)$ and

(12) $R_{1}=R$ and $R_{2}$ is an arbitrary subalgebra of $R$,

(13) $R_{1} \cong F^{2}$ and $R_{2} \cong F_{0}$,

(14) $R_{1} \cong\left(\begin{array}{cc}F & F \\ 0 & 0\end{array}\right)$ and $R_{2} \cong F$,

(15) $R_{1} \cong F \times F_{0}$ and $R_{2} \cong F$,

(16) $R_{1} \cong F^{2}$ and $R_{2} \cong F \times F_{0}$,

(17) $R_{1} \cong F^{2}$ and $R_{2} \cong\left(\begin{array}{cc}F & F \\ 0 & 0\end{array}\right)$,

(18) $R_{1} \cong\left(\begin{array}{cc}F & F \\ 0 & 0\end{array}\right)$ and $R_{2} \cong F \times F_{0}$.

Proof. We can suppose without loss of generality that $\operatorname{dim}_{F}\left(R_{1}\right) \geq \operatorname{dim}_{F}\left(R_{2}\right)$.

(I) Let $m=0$ and $R=A$. Then $m=0, R$ is a local algebra and either $R / J(R) \cong F$ or $J(R)=0$ and $R$ is a field. Note that the case $\operatorname{dim}_{F}(R / J(R))=2$ is excluded, since $J(R) / J^{2}(R)$ should be an $R / J(R)$-vector space and hence $2 \mid \operatorname{dim}_{F}(R)$.

If $J(R)=0$, then $R$ is a field and its subfields of $F$-dimension is either 1 or 3 . Hence either $R_{1}=R$ or $R_{2}=R$. 
If $R / J(R) \cong F$ and $J^{2}(R)=0$, then $R \cong F\left[x_{1}, x_{2}\right] /\left\langle x_{1}^{2}, x_{1} x_{2}, x_{2}^{2}\right\rangle$. Hence we have one of the possibilities (1)-(5).

If $R / J(R) \cong F$ and $J^{2}(R) \neq 0$, then $J^{3}(R)=0$ and there exists a basis $1, j, j^{2}$ of $R_{F}$, where $j \in J(R)$ and $j^{2} \in J^{2}(R)$. Hence $R \cong F[x] /\left\langle x^{3}\right\rangle$. Now we have one of the possibilities (6)-(8).

(II) If $m=1, \operatorname{dim}_{F}(R / J(R)) \geq 2$ and so $\operatorname{dim}_{F}(J(R)) \leq 1$.

If $J(R)=0$, then clearly, $R \cong F \times G$, where $G$ is a field of $F$-dimension 2 and we have one of the possibilities (1)-(3).

If $J(R) \neq 0$, then $R / J(R) \cong F \times F$ and $\operatorname{dim}_{F}(J(R))=1$. As $J(R)$ is an $F \times F$ module, we get that $R \cong\left(\begin{array}{cc}F & F \\ 0 & F\end{array}\right)$. Note that $R \cong\left(\begin{array}{ll}F & 0 \\ F & F\end{array}\right)$. Hence we have one of the possibilities (4)-(10).

(III) This is clear.

(IV) We assume that $m=0$. Then $R=J(R)$ for a local algebra $A$ of the dimension 4. If $J^{2}(R)=0$, then $R \cong F_{0}^{3}$. So (a) is clear. If $J^{2}(R) \neq 0$ and $J^{3}(R)=0$, then we get $\operatorname{dim}_{F}\left(J(R) / J^{2}(R)\right)=2$ and $\operatorname{dim}_{F}\left(J^{2}(R) / J^{3}(R)\right)=1$. Then there exists a basis $\left\{j_{1}, j_{2}, j_{3}\right\}$ of $R$ and elements $a_{k l} \in F, k, l \in\{1,2\}$ such that $j_{1}, j_{2} \in J(R) \backslash J^{2}(R)$, $j_{3} \in J^{2}(R)$ and $j_{k} j_{l}=a_{k l} j_{3}$ for all $k, l \in\{1,2\}$. Now it is easy to see that $A \cong S\left(\left(a_{k l}\right)\right)$ and so we have one of the possibilities (1)-(6).

If $J^{3}(R) \neq 0$, then $J^{4}(R)=0$ and $\operatorname{dim}_{F}\left(J(R) / J^{2}(R)\right)=\operatorname{dim}_{F}\left(J^{2}(R) / J^{3}(R)\right)=$ $\operatorname{dim}_{F}\left(J^{3}(R)\right)=1$ and there is a base $\left\{j, j^{2}, j^{3}\right\}$ of $R$. Hence $R=R_{1} \cong x F[x] /\left\langle x^{4}\right\rangle$ and $R_{2}$ is an arbitrary (chain) subalgebra of $R$.

(V) Let $m=1$ and $R \neq A$. Then either $e_{0} J(R) \neq 0$ or $J(R) e_{0} \neq 0$ and $A / J(R) \cong$ $F \times G$ where $G$ is a field and $\operatorname{dim}_{F}(G) \leq 2$. Let $G \cong F$. First, moreover, suppose that $J^{2}(R)=0$ and we will discuss cases of $\mu(J(R))$, denote $\left\{j_{1}, j_{2}\right\}$ a base of $J(R)$ such that $j_{k} \in e_{a_{k}} J(R) e_{b_{k}}$ for a suitable $a_{k}$ and $b_{k}$. Note that either the first row or the first column is nonzero and $j_{k} j_{l}=0$. Hence it remains to express multiplications $e_{1} j_{k}$ and $j_{k} e_{1}$, which follows immediately from the occurrence of $j_{k}$ in $e_{a_{k}} J e_{b_{k}}$.

If $\mu(J(R))=\left(\begin{array}{ll}1 & 0 \\ 0 & 1\end{array}\right)$, then $j_{1} \in e_{0} J(R) e_{0}, j_{2} \in e_{1} J(R) e_{1}, e_{1} j_{1}=j_{1} e_{1}=0$ and $e_{1} j_{2}=j_{2} e_{1}=j_{2}$. Thus $R \cong F_{0} \times F[x] /\left\langle x^{2}\right\rangle$ and one of the possibilities (1)-(5) holds true. 


$$
\begin{gathered}
\text { If } \mu(J(R))=\left(\begin{array}{ll}
0 & 1 \\
0 & 1
\end{array}\right) \text {, then } j_{1} \in e_{0} J(R) e_{1}, j_{2} \in e_{1} J(R) e_{1}, e_{1} j_{1}=0, j_{1} e_{1}=j_{1} \\
\text { and } e_{1} j_{2}=j_{2} e_{1}=j_{2} \text {. Hence } R \cong\left\{\left(\begin{array}{lll}
0 & 0 & b \\
0 & a & c \\
0 & 0 & a
\end{array}\right) \mid a, b, c \in F\right\} \text {. Hence one of the }
\end{gathered}
$$
possibilities (6)-(12) holds true.

$$
\begin{gathered}
\text { If If } \mu(J(R))=\left(\begin{array}{ll}
1 & 0 \\
1 & 0
\end{array}\right) \text {, then } j_{1} \in e_{0} J(R) e_{0}, j_{2} \in e_{1} J(R) e_{0}, e_{1} j_{1}=e_{1} j_{2}= \\
j_{1} e_{1}=0 \text { and } j_{2} e_{1}=j_{2} \text {. Thus } R \cong\left(\begin{array}{ccc}
F & 0 & F \\
0 & 0 & F \\
0 & 0 & 0
\end{array}\right) \text { and one of the possibilities }
\end{gathered}
$$

(13)-(19) holds true

$$
\begin{gathered}
\text { If } \mu(J(R))=\left(\begin{array}{ll}
1 & 1 \\
0 & 0
\end{array}\right) \text {, then } j_{1} \in e_{0} J(R) e_{0} \text { and } j_{2} \in e_{0} J(R) e_{1} \text {. Hence } e_{1} j_{1}= \\
j_{2} e_{1}=j_{1} e_{1}=0 \text { and } e_{1} j_{2}=j_{2} \text {, and so } R \cong\left(\begin{array}{ccc}
F & 0 & 0 \\
0 & 0 & 0 \\
F & F & 0
\end{array}\right) \text {. Similarly as in (c), we }
\end{gathered}
$$

need not to describe $R_{1}$ and $R_{2}$, since the case is antiisomorphic to the case (d).

If $\mu(J(R))=\left(\begin{array}{ll}0 & 1 \\ 1 & 0\end{array}\right)$, then $j_{1} \in e_{0} J(R) e_{1}, j_{2} \in e_{1} J(R) e_{0}, e_{1} j_{1}=j_{2} e_{1}=0$, $j_{1} e_{1}=j_{1}$ and $e_{1} j_{2}=j_{2}$, which implies that $R \cong\left\{\left(\begin{array}{ccc}a & b & 0 \\ 0 & 0 & c \\ 0 & 0 & a\end{array}\right) \mid a, b, c \in F\right\}$.

Hence one of the possibilities (20)-(26) holds true.

The the possibilities (27)-(29) are clear.

Finally assume that $J^{2}(R) \neq 0$. Then $\operatorname{dim}_{F}\left(J(R) / J^{2}(R)\right)=\operatorname{dim}_{F}\left(J^{2}(R)\right)=1$ and there exist a base $j_{1}, j_{2}$ of $J(R)$ such that $j_{1}^{2}=j_{2} \in J^{2}(R)$. Note that $j_{1} \in$ $e_{0} J(R) e_{0}$ since $j_{1}^{2} \neq 0$ and $e_{0} J(R) \neq 0$. Hence we get $R \cong F \times\left(x F[x] /\left\langle x^{3}\right\rangle\right)$ and the possibilities (30)-(22) are clear.

(VI) Let $m=2$ and $R \neq A$. Clearly, $A / J(R) \cong F^{3}$ and either $e_{0} J(R) \neq 0$ or $J(R) e_{0} \neq 0$. As a similar calculation in (V), we get that:

If $e_{0} J(R) \neq 0$ then $R \cong F^{2} \times F_{0}$ and one of the possibilities (1)-(4) holds.

If either $e_{0} J(R) e_{1} \neq 0$ or $e_{0} J(R) e_{2} \neq 0$, then $R \cong F \times\left(\begin{array}{cc}F & 0 \\ F & 0\end{array}\right)$ and one of the possibilities (5)-(11) holds. 
ON FINITE DIMENSIONAL ALGEBRAS WHICH ARE SUMS OF TWO SUBALGEBRAS 143

If either $e_{1} J(R) e_{0} \neq 0$ or $e_{2} J(R) e_{0} \neq 0$, then $R \cong F \times\left(\begin{array}{cc}F & F \\ 0 & 0\end{array}\right)$ and one of the possibilities (12)-(18) holds.

We conclude the paper with the following problems for further studies.

Problem 1. Describe all finite dimensional algebras over a field which are sums of two subalgebras.

Problem 2. Describe all finite dimensional algebras over a field which are sums of two nilpotent subalgebras.

Problem 3. Describe all finite dimensional algebras which are sums of two matrix algebras over a field.

Acknowledgements: First author wishes to thank Prof. Edmund Puczylowski for his valuable discussions during the preparation of this paper. We warmly thank the referee for carefully reading and commenting a first version of this article.

\section{References}

[1] K. I. Beidar and A. V. Mikhalev, Generalized polynomial identities and rings that are sums of two subrings, Algebra i Logika, 34(1) (1995), 3-11.

[2] L. A. Bokut, Imbeddings into simple associative algebras, Algebra i Logika, 15(2) (1976), 117-142.

[3] B. Felzenszwalb, A. Giambruno and G. Leal, On rings which are sums of two PI-subrings: a combinatorial approach, Pacific J. Math., 209(1) (2003), 17-30.

[4] O. H. Kegel, Zur Nilpotenz gewisser assoziativer Ringe, Math. Ann., 149 (1962/63), 258-260.

[5] O. H. Kegel, On rings that are sums of two subrings, J. Algebra, 1 (1964), 103-109.

[6] A. V. Kelarev, A sum of two locally nilpotent rings may be not nil, Arch. Math. (Basel), 60 (1993), 431-435.

[7] M. Kepczyk, Note on algebras which are sums of two PI subalgebras, J. Algebra Appl., 14 (2015), 1550149 (10 pp).

[8] M. Kepczyk, A note on algebras that are sums of two subalgebras, Canad. Math. Bull., 59 (2016), 340-345.

[9] M. Kepczyk, A ring which is a sum of two PI subrings is always a PI ring, Israel J. Math., 221(1) (2017), 481-487. 
[10] M. Kepczyk and E. R. Puczylowski, On radicals of rings which are sums of two subrings, Arc. Math. (Basel), 66(1) (1996), 8-12.

[11] M. Kepczyk and E. R. Puczylowski, Rings which are sums of two subrings, Ring Theory (Miskolc, 1996), J. Pure App. Algebra, 133(1-2) (1998), 151-162.

[12] M. Kepczyk and E. R. Puczylowski, Rings which are sums of two subrings satisfying polynomial identities, Comm. Algebra, 29(5) (2001), 2059-2065.

[13] M. Kepczyk and E. R. Puczylowski, On the structure of rings which are sums of two subrings, Arc. Math. (Basel), 83(5) (2004), 429-436.

[14] G. Köthe, Die Struktur der Ringe, deren Restklassenring nach dem Radikal vollstanding irreduzibel ist., Math. Z., 32 (1930), 161-186.

[15] A. Smoktunowicz, On some results related to Köthe's conjecture, Serdica Math. J., 27 (2001), 159-170.

[16] A. Smoktunowicz, A simple nil ring exists, Comm. Algebra, 30(1) (2002), 2759.

[17] B. Stenström, Rings of Quotients: Die Grundlehren der Mathematischen Wissenschaften, Band 217, An introduction to methods of ring theory, SpringerVerlag, New York-Heidelberg, 1975.

M. Tamer Koşan (Corresponding Author)

Department of Mathematics

Faculty of Sciences

Gazi University

Ankara, Turkey

e-mail: mtamerkosan@gazi.edu.tr, tkosan@gmail.com

\section{Jan Žemlička}

Department of Algebra

Charles University in Prague

Faculty of Mathematics and Physics

Sokolovská 83, 18675 Praha 8, Czech Republic

e-mail: zemlicka@karlin.mff.cuni.cz 